\title{
Monte Carlo studies of supersymmetric matrix quantum mechanics with sixteen supercharges at finite temperature
}

\author{
Konstantinos N. Anagnostopoulos ${ }^{1} *$ Masanori Hanada $^{2}$ 丹 Jun Nishimura 3,4 and Shingo Takeuchi 4 \\ ${ }^{1}$ Physics Department, National Technical University of Athens, Zografou Campus, GR-15780 Athens, Greece \\ ${ }^{2}$ Theoretical Physics Laboratory, RIKEN Nishina Center, 2-1 Hirosawa, Wako, Saitama 351-0198, Japan \\ ${ }^{3}$ High Energy Accelerator Research Organization (KEK), Tsukuba 305-0801, Japan \\ ${ }^{4}$ Department of Particle and Nuclear Physics, School of High Energy Accelerator Science, \\ Graduate University for Advanced Studies (SOKENDAI), Tsukuba 305-0801, Japan
}

(Dated: July, 2007; preprint: RIKEN-TH-112, KEK-TH-1165)

\begin{abstract}
We present the first Monte Carlo results for supersymmetric matrix quantum mechanics with sixteen supercharges at finite temperature. The recently proposed non-lattice simulation enables us to include the effects of fermionic matrices in a transparent and reliable manner. The internal energy nicely interpolates the weak coupling behavior obtained by the high temperature expansion, and the strong coupling behavior predicted from the dual black hole geometry. The Polyakov line takes large values even at low temperature suggesting the absence of a phase transition in sharp contrast to the bosonic case. These results provide highly non-trivial evidences for the gauge/gravity duality.
\end{abstract}

PACS numbers: 11.25.-w; 11.25.Sq

Introduction. - In the last decade, we have witnessed the increasing importance of large- $N$ gauge theories in theoretical particle physics. For instance, the holographic principle, which was inspired originally by the Bekenstein-Hawking formula for the black hole entropy, has now been given a concrete manifestation as a conjectured duality between the strongly coupled large- $N$ gauge theory and the weakly coupled supergravity. The best understood example is the AdS/CFT correspondence [1], but there are numerous extensions to non-conformal field theories as well. In particular, large- $N$ gauge theories in low dimensions have been studied intensively at finite temperature, which revealed intriguing connections to the black hole thermodynamics $[2, \underline{3}, \underline{4}, \underline{5}, \underline{6}]$.

Large- $N$ gauge theories in low dimensions also play an important role in formulating superstring/ $\mathrm{M}$ theories non-perturbatively based on the idea of matrix models, which was successful in the case of non-critical strings. For instance, it is conjectured that critical string/M theories can be formulated in terms of matrix models, which can be formally obtained by dimensionally reducing $\mathrm{U}(N)$ super Yang-Mills theory in ten dimensions to $D=0,1,2$ dimensions. In particular, the $D=1$ case [7] corresponds to the M Theory [8], which is a hypothetical eleven-dimensional theory proposed to understand the dualities among all the known superstring theories in ten dimensions.

In order to confirm these conjectures or to make use of them, it is clearly important to study large- $N$ gauge theories from first principles. Monte Carlo simulation is expected to be very useful for that purpose. In particular, totally reduced models 9 (the $D=0$ case) have been studied in refs. 10, 11, 12, 13, 14]. The complex Pfaffian, which appears from integration over the fermionic matrices, causes a technical obstacle in numerical simulation, which may be overcome by a new method proposed in ref. [14]. In fact, the phase of the Pfaffian is speculated to induce the spontaneous breaking of $\mathrm{SO}(10)$ symmetry down to $\mathrm{SO}(4)$, a scenario for the dynamical generation of four-dimensional space-time [15] suggested from the Gaussian expansion method [16].

In the $D=1$ case, some sort of "discretization" is needed in order to put the theory on computer. Given the well-known problems with the conventional lattice discretization, three of the authors (M.H., J.N. and S.T.) have proposed a non-lattice simulation method [17], which is useful for studying supersymmetric quantum mechanics. The crucial point was that the gauge symmetry is almost trivial in $1 \mathrm{~d}$, and therefore, we can fix the gauge completely. This allows us to introduce a Fourier mode cutoff $\Lambda$ without violating the gauge symmetry. In the bosonic case the new method reproduced the lattice results in the continuum limit. In the SUSY case with four supercharges, it reproduced the results of the high temperature expansion in the continuum. The same model has been studied in ref. [18] by the lattice approach using a simple lattice action and a more complicated lattice action preserving half of SUSY.

In this work we apply the non-lattice simulation method to the most interesting case with sixteen supercharges. We have reduced the computational effort considerably by introducing pseudo-fermions based on the idea of the Rational Hybrid Monte Carlo (RHMC) algorithm [19] in the way described in ref. [18].

As discussed in ref. 17], our action is nothing but the gauge-fixed action in the continuum except for having a Fourier mode cutoff. Supersymmetry, which is mildly broken by the cutoff, is shown to be restored much faster than the continuum limit is achieved. In fact, the continuum limit is also approached faster than one would naively expect from the number of degrees of freedom. This is understandable from the fact that the modes 
above the cutoff are naturally suppressed by the kinetic term. A further (albeit technical) advantage of our formulation is that we can implement the Fourier acceleration, which eliminates the critical slowing down completely [20], without extra cost since we are dealing with Fourier modes directly. We consider that all the theoretical and technical merits of the present approach compensate the superficial increase in the computational effort by the factor of $\mathrm{O}(\Lambda)$ compared to the lattice approach with the same number of degrees of freedom.

Simulation techniques. - The model can be obtained formally by dimensionally reducing 10d super Yang-Mills theory to $1 \mathrm{~d}$. The action is given by

$$
\begin{aligned}
S= & \frac{1}{g^{2}} \int_{0}^{\beta} d t \operatorname{tr}\left\{\frac{1}{2}\left(D_{t} X_{i}\right)^{2}-\frac{1}{4}\left[X_{i}, X_{j}\right]^{2}\right. \\
& \left.+\frac{1}{2} \psi_{\alpha} D_{t} \psi_{\alpha}-\frac{1}{2} \psi_{\alpha}\left(\gamma_{i}\right)_{\alpha \beta}\left[X_{i}, \psi_{\beta}\right]\right\},
\end{aligned}
$$

where $D_{t}=\partial_{t}-i[A(t), \cdot]$ represents the covariant derivative with the gauge field $A(t)$ being an $N \times N$ Hermitian matrix. It can be viewed as a one-dimensional $\mathrm{U}(N)$ gauge theory with adjoint matters. The bosonic matrices $X_{i}(t)(i=1, \cdots, 9)$ come from spatial components of the 10d gauge field, while the fermionic matrices $\psi_{\alpha}(t)(\alpha=1, \cdots, 16)$ come from a Majorana-Weyl spinor in 10d. The $16 \times 16$ matrices $\gamma_{\mu}$ in (1) act on spinor indices and satisfies the Euclidean Clifford algebra $\left\{\gamma_{i}, \gamma_{j}\right\}=2 \delta_{i j}$. We impose periodic and anti-periodic boundary conditions on the bosons and fermions, respectively. The extent $\beta$ in the Euclidean time direction then corresponds to the inverse temperature $\beta \equiv 1 / T$. The parameter $g$ in (11) can always be scaled out by an appropriate rescaling of the matrices and the time coordinate $t$. We take $g=\frac{1}{\sqrt{N}}$ without loss of generality.

We take the static diagonal gauge $A(t)=$ $\frac{1}{\beta} \operatorname{diag}\left(\alpha_{1}, \cdots, \alpha_{N}\right)$, where $\alpha_{a}$ can be chosen to satisfy the constraint $\max _{a}\left(\alpha_{a}\right)-\min _{a}\left(\alpha_{a}\right) \leq 2 \pi$ by using the large gauge transformation with a non-zero winding number. We have to add to the action a term

$$
S_{\mathrm{FP}}=-\sum_{a<b} 2 \ln \left|\sin \frac{\alpha_{a}-\alpha_{b}}{2}\right|,
$$

which appears from the Faddeev-Popov procedure, and the integration measure for $\alpha_{a}$ is taken to be uniform.

We make a Fourier expansion

$$
X_{i}^{a b}(t)=\sum_{n=-\Lambda}^{\Lambda} \tilde{X}_{i n}^{a b} \mathrm{e}^{i \omega n t} ; \psi_{\alpha}^{a b}(t)=\sum_{r=-\lambda}^{\lambda} \tilde{\psi}_{\alpha r}^{a b} \mathrm{e}^{i \omega r t},
$$

where $\lambda \equiv \Lambda-1 / 2$. The indices $n$ and $r$ take integer and half-integer values, respectively, corresponding to the imposed boundary conditions. Introducing a shorthand notation

$$
\left(f^{(1)} \cdots f^{(p)}\right)_{n} \equiv \sum_{k_{1}+\cdots+k_{p}=n} f_{k_{1}}^{(1)} \cdots f_{k_{p}}^{(p)}
$$

we can write the action (11) as $S=S_{\mathrm{b}}+S_{\mathrm{f}}$, where

$$
\begin{aligned}
S_{\mathrm{b}}= & N \beta\left[\frac{1}{2} \sum_{n=-\Lambda}^{\Lambda}\left(n \omega-\frac{\alpha_{a}-\alpha_{b}}{\beta}\right)^{2} \tilde{X}_{i,-n}^{b a} \tilde{X}_{i n}^{a b}\right. \\
& \left.-\frac{1}{4} \operatorname{tr}\left(\left[\tilde{X}_{i}, \tilde{X}_{j}\right]^{2}\right)_{0}\right] \\
S_{\mathrm{f}}= & \frac{1}{2} N \beta \sum_{r=-\lambda}^{\lambda}\left[i\left(r \omega-\frac{\alpha_{a}-\alpha_{b}}{\beta}\right) \tilde{\bar{\psi}}_{\alpha r}^{b a} \tilde{\psi}_{\alpha r}^{a b}\right. \\
& \left.-\left(\gamma_{i}\right)_{\alpha \beta} \operatorname{tr}\left\{\tilde{\bar{\psi}}_{\alpha r}\left(\left[\tilde{X}_{i}, \tilde{\psi}_{\beta}\right]\right)_{r}\right\}\right] .
\end{aligned}
$$

The fermionic action $S_{\mathrm{f}}$ may be written in the form $S_{\mathrm{f}}=\frac{1}{2} \mathcal{M}_{A \alpha r ; B \beta s} \tilde{\psi}_{\alpha r}^{A} \tilde{\psi}_{\beta s}^{B}$, where we have expanded $\tilde{\psi}_{\alpha r}=$ $\sum_{A=1}^{N^{2}} \tilde{\psi}_{\alpha r}^{A} t^{A}$ in terms of $\mathrm{U}(N)$ generators $t^{A}$. Integrating out the fermionic variables, one obtains the Pfaffian $\operatorname{Pf} \mathcal{M}$, which is complex in general. However, we observe that it is actually real positive with high accuracy in the temperature regime studied in the present work. Hence we can replace it by $|\operatorname{Pf} \mathcal{M}|=\operatorname{det}\left(\mathcal{D}^{1 / 4}\right)$, where $\mathcal{D}=\mathcal{M}^{\dagger} \mathcal{M}$.

The trick of the RHMC algorithm is to use the rational approximation $x^{-1 / 4} \simeq b_{0}+\sum_{k=1}^{Q} \frac{a_{k}}{x+b_{k}}$, which has sufficiently small relative error within a certain range required by the system to be simulated. (The real positive parameters $a_{k}$ and $b_{k}$ can be obtained by a code 21] based on the Remez algorithm.) Then the Pfaffian is replaced by $|\operatorname{Pf} \mathcal{M}|=\int d F d F^{*} \mathrm{e}^{-S_{\mathrm{PF}}}$, where

$$
S_{\mathrm{PF}}=b_{0} F^{*} F+\sum_{k=1}^{Q} a_{k} F^{*}\left(\mathcal{D}+b_{k}\right)^{-1} F,
$$

using the auxiliary complex variables $F$, which is called the pseudo-fermions.

We apply the usual HMC algorithm to the whole system as described in ref. [17], except that now we introduce the momentum variables conjugate to the pseudofermions $F$ as well as the bosonic matrices $\tilde{X}_{i}$ and the gauge variables $\alpha_{a}$. When we solve the auxiliary classical Hamiltonian dynamics, it is important to apply the Fourier acceleration 20] to the pseudo-fermions $F$ and the bosonic matrices $\tilde{X}_{i}$. The main part of the computation comes from solving a linear system $\left(\mathcal{D}+b_{k}\right) \chi=$ $F \quad(k=1, \cdots, Q)$. We solve the system for the smallest $a_{k}$ using the conjugate gradient method, which reduces the problem to the iterative multiplications of $\mathcal{M}$ to a pseudo-fermion field, each of which requires $\mathrm{O}\left(\Lambda^{2} N^{3}\right)$ arithmetic operations if implemented carefully. The solution for larger $b_{k}$ 's can be obtained as by-products using the idea of the multi-mass Krylov solver [22]. This avoids the factor of $Q$ increase of the computational effort.

Infrared instability. - Since the integration domain for the bosonic matrices is non-compact, the convergence of the partition function is not obvious. In par- 
ticular, there exists a potential danger in the flat direction corresponding to commuting matrices. Such an issue has been addressed in the totally reduced model $[10,11,15,23]$. In the present $D=1$ case, let us expand the cutoff theory (5) around the commuting background $\tilde{X}_{i 0}=\operatorname{diag}\left(x_{i 1}, \cdots, x_{i N}\right)$ and consider the effective action for the moduli parameters $x_{i a}$ and $\alpha_{a}$. When both $T$ and all of $\left|x_{i a}-x_{i b}\right|$ are large, the fluctuations become very massive, and the one-loop approximation is justified. We can easily obtain

$$
W_{1-\mathrm{loop}}=\sum_{a<b} 4 \log \left(\frac{\prod_{n}\left\{\left(2 \pi n-\alpha_{a b}\right)^{2}+\left(\beta x_{a b}\right)^{2}\right\}}{\prod_{r}\left\{\left(2 \pi r-\alpha_{a b}\right)^{2}+\left(\beta x_{a b}\right)^{2}\right\}}\right),
$$

where we have defined $\alpha_{a b} \equiv \alpha_{a}-\alpha_{b}$ and $x_{a b} \equiv$ $\sqrt{\left(x_{i a}-x_{i b}\right)^{2}}$. In eq. (7) we have omitted terms independent of $x_{a b}$, which actually vanish in the $\Lambda \rightarrow \infty$ limit. When $x_{a b} \ll T$ and $\alpha_{a b} \ll 2 \pi$, the $n=0$ term dominates and yields a logarithmic attractive potential $W_{1-\text { loop }} \simeq \sum_{a<b} 4 \log \left\{\left(\alpha_{a b}\right)^{2}+\left(\beta x_{a b}\right)^{2}\right\}$ among $\alpha_{a}$ and among $x_{i a}$. This agrees with the well-known result in the bosonic IKKT model, which describes the high temperature limit of the present model. In fact one obtains $x_{a b} \sim T^{1 / 4}$ according to the high temperature expansion (HTE) 24]. On the other hand, when $T \ll x_{a b} \ll 2 \pi \Lambda T$, the denominator and the numerator in eq. (7) cancel each other almost completely. This implies the existence of an instability.

As $T$ is lowered, the instability region approaches the peak at $x_{a b} \sim T^{1 / 4}$ representing the high temperature behavior. However, since we have a sum over all the pairs of indices $(a, b)$, a tiny difference between the denominator and the numerator is enhanced by the factor of $N^{2}$. Then it follows that the lower edge of the instability region gets multiplied by $N$. We found empirically that the instability can be avoided by taking $N \gtrsim \frac{6}{T}$, which is consistent with the above considerations.

At low temperature, one also has to worry about the finite $\Lambda$ effects. In the case of energy, they are negligible for $\Lambda \gtrsim \frac{2}{T}$, whereas for the other observables studied in this paper, we need twice as large $\Lambda$.

Results. - Fig. 11 shows the average of the "extent of space" $R^{2}=\frac{1}{N \beta} \int d t \operatorname{tr}\left(X_{i}\right)^{2}$. The instability mentioned above can be probed by the divergence of this quantity. We obtain stable results at sufficiently large $N$.

In fig. 2 we plot the absolute value of the Polyakov line, which is the order parameter for the SSB of the U(1) symmetry. Unlike in the bosonic case [5, 25, 26], where the SSB occurs around $T \simeq 1$, the Polyakov line is not small even at low $T$. This implies that there is no phase transition in the SUSY case, as predicted by the gauge/gravity correspondence [3, $[6]$. We find that the Polyakov line can be fitted nicely to

$$
\langle|P|\rangle=\exp \left(-\frac{a}{T}+b\right)
$$

a characteristic behavior in a deconfined theory.

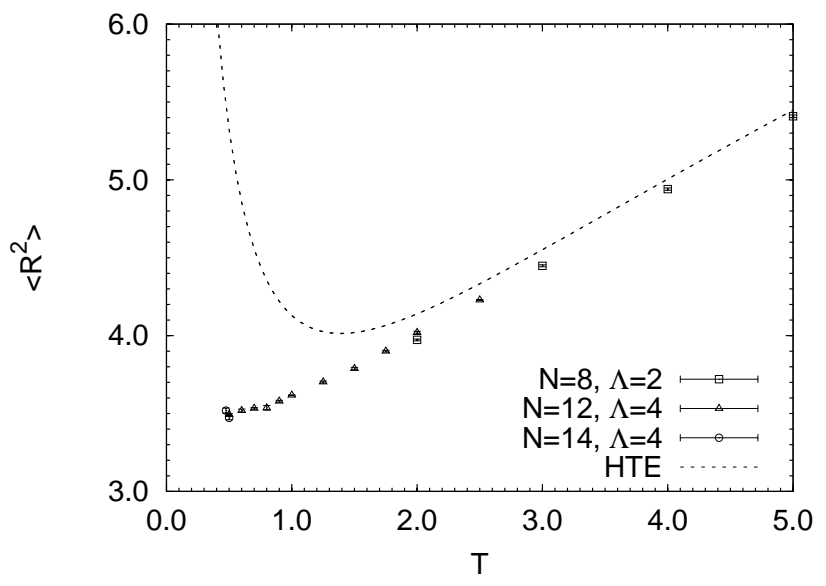

FIG. 1: The "extent of space" is plotted against temperature. The dashed line represents the result obtained by HTE up to the next leading order for $N=8[24]$.

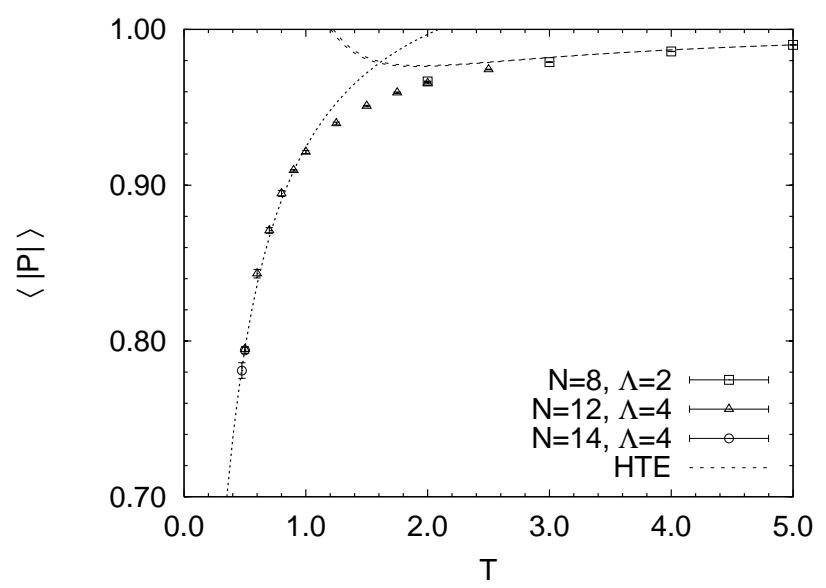

FIG. 2: The absolute value of the Polyakov line is plotted against temperature. The dashed line represents the result obtained by HTE up to the next leading order for $N=8$ [24]. The dotted line represents a fit to eq. (8) with $a=0.15$ and $b=0.072$.

In fig. 3 we plot the internal energy defined by $E=$ $\frac{\partial}{\partial \beta}(\beta \mathcal{F})$, where $\mathcal{F}$ is the free energy of the system. In practice, we calculate it using a formula, which follows from a simple scaling argument [18]. In our case it reads $E=-3 T\left[\left\langle S_{\mathrm{b}}\right\rangle-\frac{9}{2}\left\{(2 \Lambda+1) N^{2}-1\right\}\right]$. Our results interpolate nicely the weak coupling behavior - calculated by the HTE up to the next leading order 24] - and the strong coupling behavior $E / N^{2}=7.4 \cdot T^{2.8}$ predicted by the gauge/gravity duality [2] from the dual black-hole geometry [27]. The power-law behavior sets in at $T \simeq 0.5$, which is reasonable since the effective coupling constant is given by $\lambda_{\text {eff }}=1 / T^{3}$ in our convention.

In ref. 4] the Gaussian expansion method was applied to the present model, and the energy obtained 
at the leading order was fitted nicely to the power law $E / N^{2}=3.2 \cdot T^{2.7}$ within $0.25 \lesssim T \lesssim 1$. Their results are in reasonable agreement with our data at $T \sim 1$, but disagree at lower temperature.

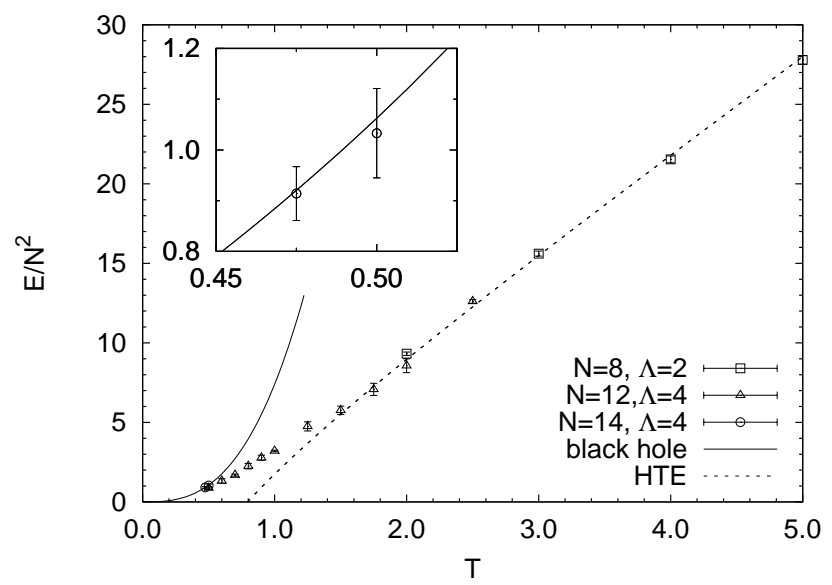

FIG. 3: The energy is plotted against temperature. The dashed line represents the result obtained by HTE up to the next leading order for $N=8$ [24]. The solid line represents the energy predicted at small $T$ by the gauge/gravity duality. The upper left panel zooms up the region, where the powerlaw behavior sets in.

Summary. - In this paper we have presented the first Monte Carlo results for the maximally supersymmetric matrix quantum mechanics, which is expected to play a very important role in string/M theories. The recently proposed non-lattice simulation together with the RHMC algorithm enabled us to study the low temperature behavior, which was not accessible by the high temperature expansion. As we lower the temperature, we observed the infrared instability, which was found to be eliminated, however, by increasing $N$. We gave a natural explanation to this phenomenon based on the one-loop effective action. Our data for the internal energy asymptote nicely to the result obtained from the dual geometry, which we consider as a highly nontrivial evidence for the gauge/gravity duality in the non-conformal case. In particular, our results suggest that the maximally supersymmetric matrix quantum mechanics exactly reproduces not only the power but also the coefficient of the power-law behavior obtained from the dual black-hole geometry.

Acknowledgments. - The authors would like to thank Shoji Hashimoto and Hideo Matsufuru for helpful suggestions concerning the RHMC simulation. The computations were carried out on supercomputers (SR11000 at KEK, SX8 at RCNP and SX7 at RIKEN) as well as on PC clusters. This work is supported by the EPEAEK programmes "Pythagoras II" and co-funded by the European Union (75\%) and the Hellenic state (25\%).
* Electronic address: konstant@mail.ntua.gr

† Electronic address: hana@riken.jp

‡ Electronic address: jnishi@post.kek.jp

$\S$ Electronic address: shingo@post.kek.jp

[1] J. M. Maldacena, Adv. Theor. Math. Phys. 2, 231 (1998).

[2] N. Itzhaki, J. M. Maldacena, J. Sonnenschein and S. Yankielowicz, Phys. Rev. D 58, 046004 (1998).

[3] J. L. F. Barbon, I. I. Kogan and E. Rabinovici, Nucl. Phys. B 544, 104 (1999).

[4] D. Kabat, G. Lifschytz and D. A. Lowe, Phys. Rev. Lett. 86, 1426 (2001); Phys. Rev. D 64, 124015 (2001).

[5] O. Aharony, J. Marsano, S. Minwalla and T. Wiseman, Class. Quant. Grav. 21, 5169 (2004).

[6] O. Aharony, J. Marsano, S. Minwalla, K. Papadodimas, M. Van Raamsdonk and T. Wiseman, JHEP 0601, 140 (2006).

[7] T. Banks, W. Fischler, S. H. Shenker and L. Susskind, Phys. Rev. D 55, 5112 (1997).

[8] E. Witten, Nucl. Phys. B 443, 85 (1995).

[9] N. Ishibashi, H. Kawai, Y. Kitazawa and A. Tsuchiya, Nucl. Phys. B 498, 467 (1997).

[10] W. Krauth, H. Nicolai and M. Staudacher, Phys. Lett. B 431, 31 (1998); W. Krauth and M. Staudacher, Phys. Lett. B 435, 350 (1998).

[11] T. Hotta, J. Nishimura and A. Tsuchiya, Nucl. Phys. B 545, 543 (1999).

[12] Z. Burda, B. Petersson and J. Tabaczek, Nucl. Phys. B 602, 399 (2001); Z. Burda, B. Petersson and M. Wattenberg, JHEP 0503, 058 (2005).

[13] J. Ambjorn, K. N. Anagnostopoulos, W. Bietenholz, T. Hotta and J. Nishimura, JHEP 0007, 011 (2000); JHEP 0007, 013 (2000).

[14] K. N. Anagnostopoulos and J. Nishimura, Phys. Rev. D 66, 106008 (2002).

[15] H. Aoki, S. Iso, H. Kawai, Y. Kitazawa and T. Tada, Prog. Theor. Phys. 99, 713 (1999).

[16] J. Nishimura and F. Sugino, JHEP 0205, 001 (2002); H. Kawai, S. Kawamoto, T. Kuroki, T. Matsuo and S. Shinohara, Nucl. Phys. B 647, 153 (2002); T. Aoyama and H. Kawai, Prog. Theor. Phys. 116, 405 (2006).

[17] M. Hanada, J. Nishimura and S. Takeuchi, arXiv:0706.1647 [hep-lat].

[18] S. Catterall and T. Wiseman, arXiv:0706.3518 [hep-lat].

[19] M. A. Clark, A. D. Kennedy and Z. Sroczynski, Nucl. Phys. Proc. Suppl. 140, 835 (2005).

[20] S. Catterall and S. Karamov, Phys. Lett. B 528, 301 (2002).

[21] M.A. Clark and A.D. Kennedy, http://www.ph.ed.ac.uk/ mike/remez, 2005.

[22] B. Jegerlehner, arXiv:hep-lat/9612014.

[23] P. Austing and J.F. Wheater, JHEP 0102, 028 (2001); JHEP 0104, 019 (2001).

[24] N. Kawahara, J. Nishimura and S. Takeuchi, in preparation.

[25] R. A. Janik and J. Wosiek, Acta Phys. Polon. B 32 (2001) 2143.

[26] N. Kawahara, J. Nishimura and S. Takeuchi, arXiv:0706.3517 [hep-th].

[27] I.R. Klebanov and A.A. Tseytlin, Nucl. Phys. B 475, 164 (1996). 